\title{
Guidelines for Robot-Human Environments in Therapy
}

\author{
Tamie Salter and Kerstin Dautenhahn \\ Adaptive Systems Research Group, School of Computer Science, \\ University of Hertfordshire, College Lane, \\ Hatfield AL10 9AB, United Kingdom \\ E-mail t.salter, k.dautenhahn@herts.ac.uk
}

\begin{abstract}
In this paper we propose guidelines for environmental conditions when using autonomous mobile robots in therapy. We investigate readings from infrared sensors on-board a mobile robot whilst the robot is maneuvering in noisy cluttered environments. Suggestions on how to structure the environment to minimise noise within the robot's sensor readings are given. Effect of different environmental conditions on the infrared readings are catalogued, including boxes and chairs. Specifically, we investigate under what conditions a method developed by Salter et al. [4, 5], which used infrared sensors to distinguish between the environment and human contact, is reliable. We also discuss related research and how the guidelines proposed here might extend past proposals for using robots in therapy situations to general robot-human environments.
\end{abstract}

\section{Introduction}

The AuRoRA project (www.aurora-project.com) is investigating how robots can be used in a therapeutic or educational context with autistic children. Work by our group has already shown encouraging results $[7,6]$. Related work includes Michaud and ThébergeTurmel [1], Wada et al. [2] and Prazak [3]. In this paper we investigate how the physical environment can affect readings of infrared sensors located on mobile robots that we have used in previous trials. Specifically we investigate the reliability of a new system developed by our group for use with robots to detect and record interaction from the children [4]. In this system Salter et al. use infrared sensors located on a robot to measure distances in the environment. The robot detects objects in the environment and avoids them so as not to come into close contact. Based on the infrared sensor readings, we used a thresholdbased method of detecting interactions, so that close contact from moving children in the environment is recorded ${ }^{1}$. This method has already been proven reliable under laboratory conditions where children's interactions have been recorded successfully whilst the robot was manuevering in an uncluttered environment [4]. Interaction levels have been used to cluster children into personality types. This clustering is therapeutically relevant since it might allow the robot in future to adapt to individual children's behaviour. In this paper, we investigate more natural conditions which might be found in real therapy situations. We investigate to what extent our method is still reliable when no children, but objects are present in the environment which might create noise in the robot's sensor data. We will systematically study under which environmental conditions, in the absence of children, interactions or noise will be registered by the robot. It is hoped that from these experiments we will be able to issue guidelines as to under what conditions our method of detecting and recording interaction via infrared sensors can be used reliably. We hope to advise how to setup the environment for robot-human interaction in a therapy context.

In the work by Salter et al. [4] they discuss how noise from the environment, e.g. a child pushing the robot against a wall, can be measured as interaction from a person when it was not. A later study carried out by Salter et al. found that in natural conditions the robot did at times get stuck against a wall causing misclassification [5]. It is theorised that misclassification will come about when the robot's manoeuvring space is such that it is forced to come into close proximity with objects so that it records interaction. We have loosely termed the extent of objects in the environment as the level of 'clutteredness', i.e. how cluttered or busy the environment is before causing

\footnotetext{
${ }^{1}$ Note: in this paper we refer to 'interaction' as any measurement that falls into this category, based on the robot's sensor readings, and regardless of whether these interactions were caused by encounters with animate or inanimate entities (e.g. children or obstacles).
} 


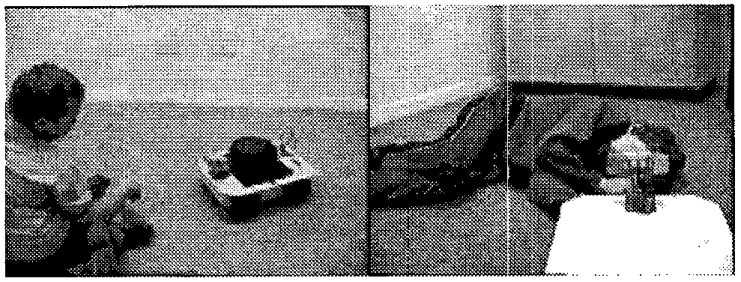

(A)

(日)

Figure 1: Pictures from video footage of Werry et al.'s work showing different therapy rooms (A) A room empty apart from 2 chairs at the back (not visible) (B) A room empty apart for a wardrobe.

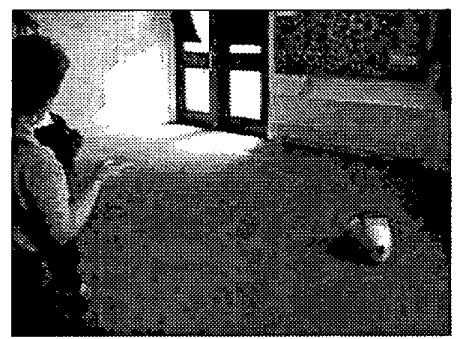

Figure 2: Picture showing a therapy session with the robot used in these experiments.

enough obstruction that interaction is recorded when none existed. Environmental effects on robot sensor readings are not only of interest in robot therapy sessions but also in the general area of human-robot interaction. Therefore we hope the work reported here can be extended to general guidelines for assessing and designing robot-human environments.

\section{Therapy Conditions}

Work with a mobile robot and children with autism carried out by Werry et al. [6] was conducted in a real therapy room at a school for autistic children. The conditions in the study were typical of what you would expect to find in specialist schools, quite often these schools will have special rooms set aside for a host of different therapies. The laboratory environment to be used in our experiments is not dissimilar from therapy conditions found at specialist schools i.e. typical therapy conditions found by Werry et al. [6]. 'The environment quite often consists of an individual child with the therapy aid and care worker(s). In such situations the environment is usually highly structured and relatively uncluttered in order to focus attention, e.g. on a few relevant stimuli. Thus in these rooms the clutteredness (other objects in the environment) is usually low. The sessions carried out by Werry et al. were conducted in a $2 \mathrm{~m}$ by $3 \mathrm{~m}$ room. The only objects in the room were two chairs, the odd piece of furniture, the robot, two experimenters, a care worker and the child (see Fig. 1). Fig. 2 shows a therapy room with a care worker, child and the mobile robot used in our experiments. However, often therapy situations provide a more natural and comfortable environment for the child, e.g. including soft play objects. It is our aim to investigate how the environment should be structured for the method developed by Salter et al. to remain reliable [4].

\section{Experimental Setup (Envi- ronmental Conditions)}

(A)

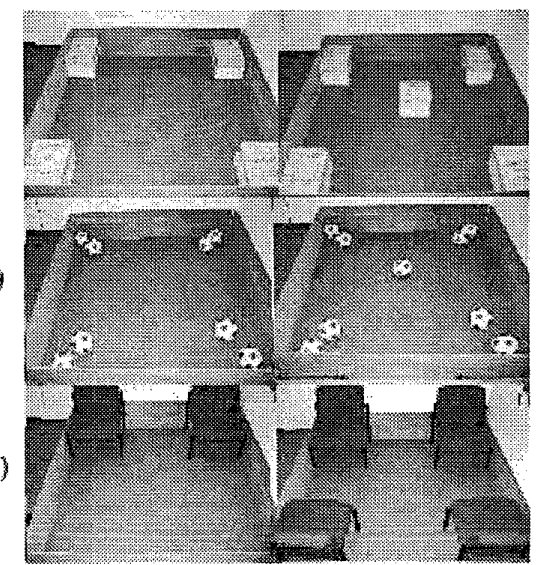

Figure 3: Pictures showing the different environmental conditions used for the experiments. The robot (seen in Fig. 2) was placed in the pen and carried out obstacle avoidance.

This room contains an arena, the 'pen' of the robot, of approximately $4 \mathrm{~m}^{2}$ and is enclosed by four shallow wooden walls. A commercially available medium sized robot was used for these experiments which has also been used in real life experiments with children $[4,5]$ (see Fig. 2) (www.wanyrobotics.com). The robot came equipped with a ring of 15 infrared sensors located around its rim; it is these sensors that are used to detect objects in the environment and also to record interactions. For the experiments, the robot was left by itself in the pen carrying out obstacle avoidance with a varying amount of objects (boxes, soft balls and 
chairs). In theory no interactions should be recorded whilst the robot is in the pen on its own as there are no humans in the pen. Therefore we will discover at what level the clutteredness of the environment causes misclassifications, i.e. the sensors record interactions when no human was present. Each experiment lasted for 5 minutes, comparable to previous trials with children and the robot. It has been checked that whilst in the pen on its own without any objects present the robot does not record any interactions.

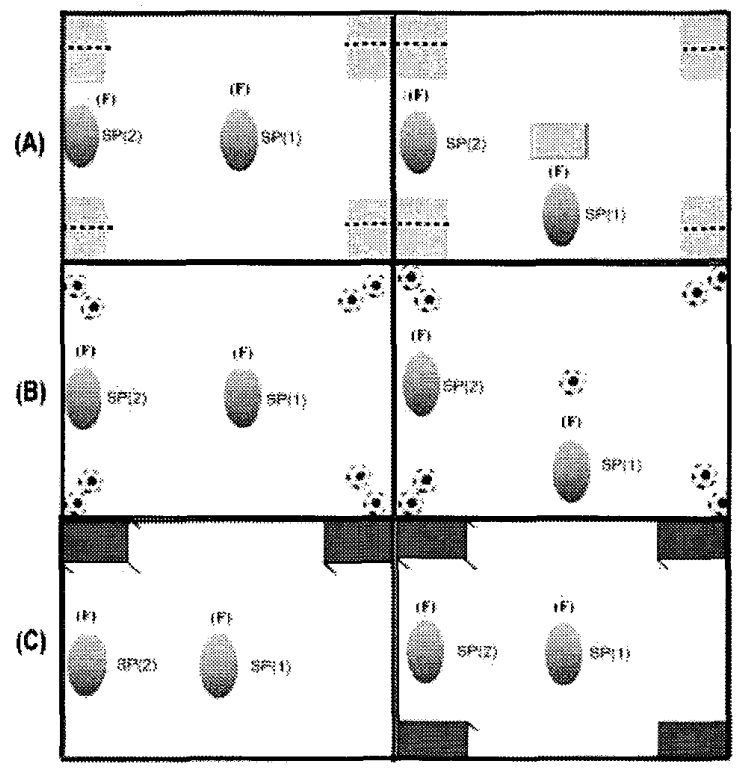

Figure 4: Pictures showing the 6 experimental setups and the two different starting points of the robot in the arena. (F) denotes the front of the robot, $\mathrm{SP}(1)$ denotes starting point $1, \mathrm{SP}(2)$ denotes starting point 2 .

\section{Experiments}

Experiments were broken down into 3 main environments: (A) contained boxes, (B) contained balls, and (C) contained chairs. Each of the 3 main environments had varying amounts of objects which created varying levels of clutteredness. Moreover, two different starting points for the robot were used. Below is a breakdown of the experiments conducted:

(A) Boxes, see Fig. 3. Each box measures $23 \mathrm{~cm}$ wide $\mathrm{x} 32 \mathrm{~cm}$ length, this means each box covers a surface area of $736 \mathrm{~cm}^{2}$ within the pen. Eight boxes - two in each corner of the pen. In total a surface area of
$5888 \mathrm{~cm}^{2}$ was covered by the eight boxes. The robot was started from the center and the side of the arena. Nine boxes - two in each corner of the pen and one in the center. In total a surface area of $6624 \mathrm{~cm}^{2}$ was covered by the nine boxes. The robot was started from the middle front and side of the arena.

(B) Soft Balls, see Fig. 3. Each ball has radius of $9.95 \mathrm{~cm}$ resulting in a surface area of $311 \mathrm{~cm}^{2}$ covered by each ball within the pen. Eight balls - two in each corner of the pen. In total a surface area of $2488 \mathrm{~cm}^{2}$ was covered by the eight balls. The robot was started from the center and the side of the arena. Nine balls - two in each corner of the pen and one in the center. In total a surface area of $2799 \mathrm{~cm}^{2}$ was covered by the nine balls. The robot was started from the middle front and side of the arena.

(C) Chairs, see Fig. 3. Each chair has four round legs. Each leg has a radius of $1.59 \mathrm{~cm}$ meaning that a surface area of $7.94 \mathrm{~cm}^{2}$ is covered by each leg. As there are four legs to each chair this gives a total surface coverage of $31.77 \mathrm{~cm}^{2}$ per chair. Note, the robot can fit under the chair's seat and between the chair's legs. Two chairs - one in each top corner of the pen. In total a surface area of $63.54 \mathrm{~cm}^{2}$ was covered. The robot was started from the center and side of the arena. Four chairs - one in each corner of the pen. In total a surface area of $127.08 \mathrm{~cm}^{2}$ was covered. The robot was started from the center and side of the arena.

Each of the 6 experimental setups was tested with 2 different starting positions (leading to 12 experimental conditions), and was repeated 5 times. In total 60 experiments were carried out, each lasting 5 minutes.

\subsection{Getting Stuck}

It has been found that in some real life situations the robot will get stuck against objects in the environment, such as against chairs or in corners of rooms, and require help from the experimenter to get free. This situation has happened in an earlier trial by Salter et al. [5] and was also a common situation in previous work by Werry et al. [6]. When getting stuck the robot's sensors show unusual readings and levels of interaction are incorrectly measured. It is hoped that our experiments will give us a greater insight into sensor readings whilst the robot is getting stuck which might ultimately lead to the robot being able to detect this situation and classify it accordingly in its sensor data. Developing a method that can distinguish between the children's interactions and the environment will be of great benefit to our work. It will be a stepping stone towards allowing our robots to react to the children's interaction differently to that 
of walls or other inanimate objects. At times during these experiments the robot got stuck either against a chair leg or against a wall. Once the robot was stuck, which can be defined by the wheels turning but the robot not moving forward, the experimenter entered the 'pen' and pulled the robot free. The experimenter approached the robot and pulled the robot back either by the tail or the top of the robot. This is how the experimenter would free the robot from being stuck in a real trial and so it was kept as close as possible to real life situations. Sometimes when the robot got stuck in real trials the child involved in the session would free the robot. Methods used by the children to free the robot range from pulling the robot by the tail to picking the robot up and walking with it to different areas in the 'pen'.

\section{Observations}

In the following we list observations from the experiments describing the robot's behaviour in more detail. Eight boxes starting point SP(1) center. Robot went round in a right hand circle without any problems.

Eight boxes starting point $\mathbf{S P}(2)$ side. Interactions are recorded at the beginning of session due to the robot coming in close contact with the box directly in front of it. Sensors record interaction first at the front left hand side of the robot then at the back sensors as the robot turns and gets out from the close contact with the boxes. Then the robot goes round in a right hand circle, again without any problems.

Nine boxes starting point SP(1) middle Front. Generally throughout the sessions the robot's behaviour appeared 'confused' by the lack of free area. This resulted at times in the robot not knowing which way to turn and so ended up going close to the boxes and recording interaction. The robot did always find a way or path to follow though.

Nine boxes starting point SP(2) side. Interactions are recorded at the beginning of session due to the robot coming in close contact with the box directly in front of it. Sensors record interaction first at the front left hand side of the robot then at the back sensors as the robot turns and gets out from the close contact with the boxes. Again generally throughout the sessions the robot seemed 'confused' by the lack of free area, did not know which way to turn and so ended up going close to the boxes and recording interaction.

Eight Balls starting point SP(1) center. Robot went in right hand circle in three of the sessions and in a left hand circle in two of the sessions with relative ease.

Eight Balls starting point SP(2) side. Generally these sessions were quite 'chaotic'. The robot got stuck in between the balls and the wall on 6 occasions. The experimenter needed to help free the robot. However in one session the robot went round in a right hand circle without getting stuck at all.

Nine Balls starting point $\mathbf{S P}(1)$ middle center. In these sessions the robot hit the balls and they rolled away. The robot nearly got stuck on several occasions but managed to free itself without the experimenter's intervention.

Nine Balls starting point $\mathbf{S P}(2)$ side. The robot traveled freely in these sessions avoiding the obstacles well.

Two chairs starting point $\mathbf{S P}(1)$ center. The robot got stuck frequently and also scraped chairs' legs on several occasions.

Two chairs starting point $\mathbf{S P ( 2 )}$ side. The robot usually hit the chair in front straight away and so got stuck and required help from the experimenter. Robot also scraped the chairs' legs.

Four chairs starting point SP(1) center. The robot got stuck and scraped chairs' legs in these sessions with greater frequency than in any other set of sessions, 16 scrapes in total.

Four chairs starting point SP(2) side. The robot usually hit the chair in the front straight away and so got stuck and required help from the experimenter. Robot got stuck frequently and scraped chairs' legs. For quantitative results of the interaction levels and number of times the robot got stuck see Fig. 5 and Fig. 6.

\section{Results}

The results are broken down into two sections, 'interaction levels' and 'getting stuck'.

\subsection{Interaction Levels}

Below are the average and standard deviation results for interaction levels measured in each of the 12 different experimental conditions. For results see Fig. 5, for the different experimental conditions see Fig. 4. Numbering on the $\mathrm{x}$ axis of the graph (Fig. 5) corresponds to the numbering of the different environmental conditions (Fig. 4).

- When there are only eight boxes and a clear maneuvering space for the robot very low levels of 
interaction are recorded, see conditions $1 \& 2$ in Fig. 5. However, adding an extra box to the arena and lowering the amount of maneuvering space caused very high interaction levels, see conditions $3 \& 4$ in Fig. 5.

- The experiments with the balls were the most 'chaotic', i.e. sometimes the balls starting rolling after being touched or hit by the robot. The five repetitions therefore showed greater variation in interaction levels (cf. conditions 6 and 7 in Fig. 5), compared to relatively small variations in other conditions (cf. conditions 4 and 5 in Fig. $5)$.

- The robot found it difficult to maneuver around chairs. This is perhaps due to the small surface area of their legs. This allows the chair legs to go undetected by the infrared sensors, ultimately resulting in the robot hitting or scraping them. Despite this, low levels of interaction were recorded. This is due to the fact that when in contact with a chair leg it is possible for only one, or two, or at times no sensors to be in contact with the legs and so only few misclassifications were recorded. See conditions $9,10,11 \& 12$ in Fig. 5.

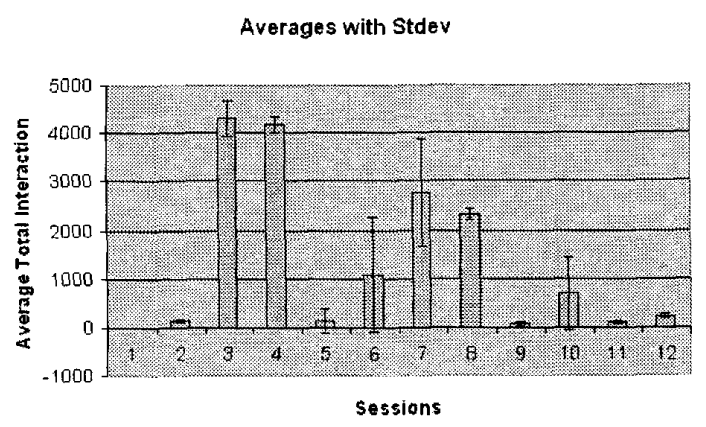

Figure 5: Graph showing the average interaction level for each of the different types of environments; error bars show standard deviation.

\subsection{Getting Stuck}

It was found that the robot got stuck in these trials. Below are the results for the number of times the robot got stuck and required the experimenter to free the robot. Results are shown for each of the $12 \mathrm{ex}-$ perimental conditions. See Fig. 6 for levels of getting stuck and for the corresponding experimental conditions see Fig. 4. Numbering on the $\mathrm{x}$ axis of the graph (Fig. 6) corresponds to the numbering of the different environmental conditions (Fig. 4). Observations show:

The robot got stuck against chairs the most. It is believed this is due to the thin diameter of a chair leg $(3.2 \mathrm{~cm})$, this allows the chair leg to be undetected by the robots sensors due to the positioning of the sensors on the robot. However, this getting stuck on chair legs did not produce high interaction levels.

Soft balls created the second highest level of getting stuck, it is believed this is not due to the object itself but the angle the object was creating with the wall. It is believed that an angle less than 90 degrees confused the robot resulting in the robot oscillating and not knowing which way to turn. This ultimately led to the robot getting stuck.

Despite boxes giving the highest interaction levels the robot did not get stuck. The solid large dimensions of the boxes seemed to allow the robot to make the correct decision about which direction to turn. Even when it came into close contact with the boxes it did not get stuck.

\section{Robot getting stuck}

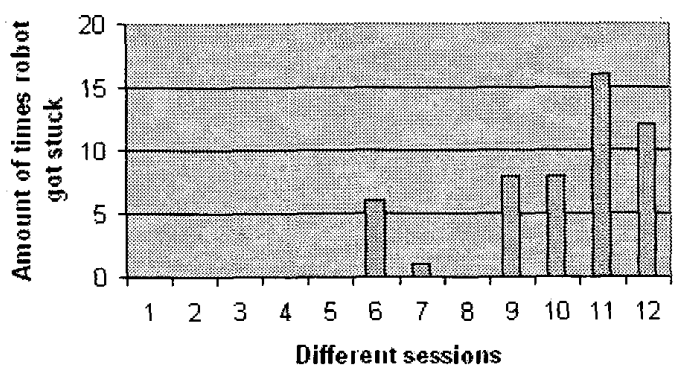

Figure 6: Graph showing the average number of times the robot got stuck in each session.

\section{Discussion}

We found that the level of objects or clutteredness of the environment does affect the robot's sensor readings. Solid objects and a low maneuvering space affected the misclassification of interaction levels more than chair legs and a low maneuvering space. This is due to the fact that chair legs have a small surface area and therefore only activate 1,2 , or no sensors at a time 
whereas large solid objects activate a high number of sensors at one time. When considering that data is being written to a file 16 times per second misclassification quickly mounts up. Getting stuck did not interfere with interaction levels as much as brushing by solid objects, cf. Fig. 5 and Fig. 6. Objects placed so as to create angles less than 90 degrees caused the robot to get stuck.

To summarise our findings of the present study: (1) Despite getting stuck on chairs more than any other object this did not cause high levels of interaction. (2) The placing the robot near to objects at the beginning of a session caused higher misclassification than placing the robot in the center. (3) Soft balls result in very random sensor readings. This appears to be because the rolling motion of the balls, and also the 45 degree angle created, seemed to cause the robot to get stuck. (4) A combination of solid objects and a low maneuvering space will result in high interaction levels.

The following are tentative design guidelines for human-robot environments in a therapeutic context where interaction is recorded based on the robot's infrared sensor data (method by Salter et al. [4]):

(a) Place the robot in the center of the room or as far away from objects as possible at the beginning of the session. (b) Keep angles in the room, above 45 degrees, as close to right angles as possible. (c) If there is a low maneuvering space for the robot try to remove unnecessary solid objects such as boxes. (d) Objects with small surface areas such as chairs may cause the robot to get stuck more but will not affect the interaction levels as much as solid objects or objects that roll. (e) Try to keep objects that roll to a minimum.

In more general robot-human interaction scenarios, not necessarily therapeutic contexts, we expect that the more cluttered the environment is the more noise will interfere with the robot's sensor data. Solid objects combined with a low manuevering space cause more noise than small objects with very small surface area regardless of manuevering space. Objects that roll, and angles less than 90 degrees cause erratic results. These conclusions are not completely unexpected or surprising as such, however our work has provided a first step toward a systematic quantitative assessment of the suitability of physical environments for robot-human interaction research. It must not be forgotten that we are attempting to develop robots that can be used by carers and laymen in therapy situations without the help of robotic experts. Any guidelines that can be given to help therapeutic carers and laymen when using robots in therapy or other- wise will be beneficial. Future studies are necessary to get a more detailed picture on requirements for robothuman interaction environments.

\section{References}

[1] F. Michaud, and C. Théberge-Turmel, "Mobile Robotic Toys and Autism", Socially Intelligent Agents - Creating Relationships with Computers and Robots, editors Dautenhahn, K. and Bond, A. and Cañamero, L. and Edmonds, B., pp. 125-132, Kluwer Academic Publishers, 2002.

[2] D. Wada, T. Shibata, T. Saito, K. Tanie, "Robot Assisted Activity for Elderly People and Nurses at a Day Service Center", IEEE IROS, pp. 1416-1421, Washington, DC, 2002.

[3] B. Prazak, A. Hochgatterer, G. Kronreif, M. Furst, "Robot Supported Play - New Possibilities for Physically Handicapped Children?!", AAATE 2003, Dublin, Ireland, 2003.

[4] T. Salter, R. te Boekhorst, K. Dautenhahn, "Detecting and analysing children's play styles with autonomous mobile robots: A case study comparing observational data with sensor readings", Proc. IAS-8, pp. 61-70, IOS Press, 2004.

[5] T. Salter, K. Dautenhahn, R. te Boekhorst, "Robots Moving out of the Laboratory - Detecting Interaction Levels and Human Contact in Noisy School Environments", Accepted for publication in Proc. IEEE RO-MAN 2004.

[6] I. Werry, K. Dautenhahn, W. Harwin, "Evaluating the Response of Children with Autism to a Robot", RESNA 2001, Rehabilitation Engineering and Assistive Technology Society of North America, John Ascuaga's Nugget Hotel, Reno, Nevada, USA 2001.

[7] B. Robins, K. Dautenhahn, R. te Boekhorst, A. Billard, "Effects of repeated exposure of a humanoid robot on children with autism", Universal Access and Assistive Technology (CWUAAT), pp. 225-236, 2004. 